\title{
Benign Submandibular Gland Neoplasm
}

National Cancer Institute

\section{Source}

National Cancer Institute. Benign Submandibular Gland Neoplasm. NCI Thesaurus. Code C4891.

A non-metastasizing neoplasm that arises from the submandibular gland. 